


\section{DE LA INEXISTENTE DISTINCIÓN ENTRE PRINCIPIOS Y REGLAS ${ }^{1}$}

\section{ADRIANA MARÍA RESTREPO OSPINA²}

REsumen: El artículo aborda el problema de la definición de los principios jurídicos en Ronald Dworkin, Robert Alexy, Manuel Atienza y Juan Ruiz Manero, como autores paradigmáticos y que han realizado aportes relevantes en la discusión sobre los principios jurídicos y su distinción respecto de las reglas. El análisis se centra en mostrar cómo cada una de estas propuestas intenta ofrecer un criterio que, desde el ámbito estructural, permita una diferenciación clara entre reglas y principios, que sirva de soporte lógico y analítico a la distinción metodológica que de ella se deriva en el terreno aplicativo: la subsunción para las reglas, y la ponderación para los principios. La revisión detallada de cada uno de estos autores lleva a concluir que dicha distinción estructural no es defendible, pues o carece de fundamentación apropiada, o supone decisiones interpretativas previas del órgano que toma la decisión.

Palabras clave: principios jurídicos, reglas, Ronald Dworkin, Robert Alexy, Manuel Atienza, Juan Ruiz Manero, diferencias estructurales.

ABSTRACT: This article addresses the issue of the definition of law's principles in Ronald Dworkin, Robert Alexy, Manuel Atienza and Juan Ruiz Manero, as paradigmatic authors who have made relevant contributions in the discussion of legal principles and their distinction from rules. The analysis focuses on showing how each one of this proposals attempts to offer a criterion that allow a clear differentiation between rules and principles from the structural

1 Este trabajo es parte del proyecto de investigación DER2016-74898-C2-1-R, "Conflictos de derechos, tipologías, razonamientos, decisiones", del Ministerio de Economía y competitividad del Gobierno español.

2 Profesora e investigadora de la Facultad de Derecho y Ciencias Políticas de la Universidad de Antioquia, Udea, calle 70 No. 52-21, Medellín, Colombia. Investigadora de la Universidad de León (España). Abogada, Magister en Filosofía y Doctoranda en Derecho de la Universidad de León (España). Correspondencia: <adrianam.restrepo@udea.edu.co>. 
point of view, which serves as a logical and analytical support to the methodological distinction in the applicative field: subsumption for rules and balancing for principles. The detailed review of each one of those authors leads to the conclusion that said structural distinction is not defensible, because of a lack of adequate foundation, or because it implies prior interpretative decisions of the agent who decides.

Keywords: Legal Principles, Rules, Ronald Dworkin, Robert Alexy, Manuel Atienza, Juan Ruiz Manero, Structural differences.

SUMARIO: I. El papel de los principios jurídicos en la teoría del derecho contemporánea: II. Los principios jurídicos desde la moral y la política en Ronald Dworkin; III. Una distinción estructural, pero centrada en el modo de cumplimiento, desde Robert Alexy; IV. Una genuina distinción estructural y sus problemas. Manuel Atienza y Juan Ruiz Manero; V. Conclusiones; VI. Fuentes.

\section{INTRODUCCIÓN}

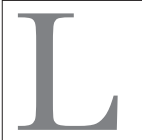

a definición de los principios jurídicos, objeto de este texto, se abordará consultando las propuestas de Ronald Dworkin, Robert Alexy, Manuel Atienza y Juan Ruiz Manero; quienes han hecho aportes relevantes en esta discusión, especialmente sobre su distinción frente a las reglas. El análisis mostrará cómo cada una de estas propuestas intenta ofrecer un criterio estructural que permita una diferenciación clara entre reglas y principios, que sirva de soporte lógico y analítico a la distinción metodológica que de ella deriva en el terreno aplicativo jurídico: la subsunción para las reglas, y la ponderación para los principios. Esta revisión detallada autores lleva a concluir que tal distinción estructural no es defendible, pues o carece de fundamentación apropiada, o supone decisiones interpretativas previas del órgano que toma la decisión.

Para ello se analizará inicialmente la importancia de la definición de los principios y su diferencia con las reglas; en segundo lugar se abordará el trabajo analítico de los textos pertinentes de los 
autores mencionados, empezando por Dworkin. Esta revisión detallada lleva a concluir que en este autor no existe criterio estructural que permita distinguir principios de reglas; a continuación se analiza conceptualmente la propuesta de Robert Alexy y sus criterios de diferenciación, enfatizando en el estructural, para concluir que lo que éste llama criterio estructural no es propiamente tal, pues se centra en el modo de cumplimiento de la regulación, no en su estructura lógica o lingüística; luego se pasa a revisar el trabajo de los profesores Atienza y Ruiz Manero, quienes en una muy detallada clasificación de los enunciados normativos sí logran ofrecer una distinción estructural importante, pero que pierde gran parte de su capacidad explicativa de la distinción entre reglas y principios, al ser directamente dependiente, de modo similar a como lo hace Alexy, de su propia definición de lo que es un principio, como un contenido interpretativo previo y configurador de la estructura misma del enunciado normativo.

I. EL PAPEL DE LOS PRINCIPIOS JURÍdicos EN LA TEORÍA DEL DERECHO CONTEMPORÁNEA

A partir de la idea de principio, entre otras, se ha configurado una manera "renovada" de entender la Constitución, que aboga abiertamente por la materialización moral del derecho, una ampliación de las capacidades de los jueces para resolver los casos controversiales -el activismo judicial- y un desplazamiento de la mítica fe en el legislador hacia una "más realista" confianza en los jueces. ${ }^{3}$ Asumiendo que los jueces poseen mayores capacidades y mejor posición (o disposición) para resolver adecuadamente los problemas que se presentan en casos difíciles, en los cuales: no hay consenso sobre los límites que el derecho impone a la decisión, no hay tales límites,

\footnotetext{
3 Juan Antonio García Amado, Pidiendo el principio. Dworkin y la teoría del derecho en serio, en línea, disponible en: <www.garciamado.es>, 2014.
} 
o los resultados de la decisión parecen inapropiados; sin que quede del todo claro si la toma de alguna decisión en estos escenarios, mediante la ponderación como su método propio, garantiza seguir moviéndose dentro del sistema jurídico o tal vez implica alguna relación exógena con otros sistemas, como el moral y/o el político.

\section{LOS PRINCIPIOS JURÍdICOS DESDE LA MORAL Y LA POLÍTICA en Ronald Dworkinw}

Desde la aparición de sus primeras críticas al positivismo, en The Model of Rules I, en 1967, la relevancia acaémica de las ideas de Dworkin fue incrementando; en la actualidad no parece posible pensar en términos de teoría jurídica sin referirse a sus ideas sobre los principios jurídicos y su papel en el derecho. Ello muestra el gran impacto de sus ideas en la teoría del derecho contemporánea, al reorientar el debate entre positivismo e iusnaturalismo ${ }^{4}$ y en la propia teoría del derecho. "Quizá el logro más importante de la obra de Dworkin sea el de haber situado los grandes problemas de la teoría jurídica dentro del ámbito de la filosofía moral y política". ${ }^{5}$

La definición de los principios jurídicos que ofrece este autor, ya clásica, dice que

[...] cuando los juristas razonan o discuten sobre derechos y obligaciones jurídicas, especialmente en aquellos casos difíciles en que nuestros problemas con tales conceptos parecen agudizarse más, echan mano de estándares que no funcionan como normas, sino que operan de modo diferente, como principios, directrices políticas y otro tipo de pautas. [...] En la mayoría de los casos usaré el término "principio" en sentido genérico, para referirme a todo el conjunto de los estándares que no son normas. ${ }^{6}$

4 Arthur Ripstein, "Dworkin,” en Ronald Dworkin, 2007.

5 Mariano Melero de la Torre, Dworkin y sus críticos, Valencia, Tirant lo Blanch, 2012, p. 11.

6 Ronald Dworkin, Los derechos en serio, Barcelona, Ariel, 1989, p. 72. 
Distingue los principios de las normas, "reglas" para Hart, ${ }^{7}$ por varias razones: porque los principios no necesariamente están escritos o pertenecen a alguna codificación, pudiendo hacerlo; porque, pueden ser tanto de carácter jurídico como morales y políticos ${ }^{8}$ son de la más alta generalidad; a diferencia de las reglas, no establecen disyuntivas, a la manera de todo o nada, sino que tienen una dimensión que aquellas no poseen: la de peso o importancia relativa para la solución de los casos.

Llamo "directriz" o "directriz política" al tipo de estándar que propone un objetivo que ha de ser alcanzado; generalmente, una mejora en algún rasgo económico, político o social de la comunidad [...] Llamo "principio" a un estándar que ha de ser observado, no porque favorezca o asegura una situación económica, política o social que se considera deseable, sino porque es una exigencia de la justicia, la equidad o alguna otra dimensión de la moralidad. ${ }^{9}$

Plantea una importante distinción interna entre principios, los principios en sentido estricto y las directrices. Los primeros expresan alguna exigencia de la moralidad; ${ }^{10}$ los segundos, algún objetivo social o político importante; siendo más relevantes para la decisión judicial los principios en sentido estricto, pues considera que las directrices son más propias para el razonamiento del legislador. ${ }^{11}$

En su debate contra el iuspositivismo, critica idea de discrecionalidad, sosteniendo que los jueces al fallar no sólo recurren a reglas, sino que también observan, especialmente en los casos difíciles, principios morales. ${ }^{12}$ Éstos también integran el derecho y permiten la existencia de una única respuesta correcta en cada

7 H. L. A Hart, The Concept of Law, 2a . ed., Oxford, Clarendon Press, 1994.

8 Dworkin, op. cit, p. 71.

9 Ronald Dworkin, Los Derechos En Serio, Barcelona, Ariel, 2012, p. 72.

10 Dworkin, op. cit., 1984, p. 72.

11 Ibidem, pp. $147 \mathrm{ss}$.

12 Ibid, p. 72 
caso. ${ }^{13}$ La teoría que mejor explique las prácticas sociales vigentes, que integran en el derecho tanto a los principios morales como a las reglas, es la mejor justificación de la decisión judicial. ${ }^{14}$

Acepta que en los casos fáciles se puede hablar de aplicación de reglas previas a casos nuevos, pero, se pregunta: “podemos decir lo mismo cuando la Suprema Corte desatiende los precedentes y ordena la desegregación en las escuelas (de los Estados Unidos) o declara ilegales procedimientos usados por la policía y condonados por los tribunales desde hace decenios?", ${ }^{15}$ casos en que, según afirma, la Corte da razones, apela a principios políticos y de justicia, sin citar precedentes ni leyes, recurriendo a "principios jurídicos fundamentales", ${ }^{16}$ utilizados por los jueces para resolver los casos difíciles.

Para sustentar la distinción planteada, el autor recurre a ejemplificar mediante casos la noción de principios. En Riggs v. Palmer, en el que el heredero mató a su abuelo, se planteaba la cuestión de si aquél debía o no recibir la herencia. En este caso, el tribunal sostuvo que:

todas las leyes, lo mismo que todos los contratos, pueden ser controladas en toda su operación y efecto por máximas generales y fundamentales del derecho consuetudinario. A nadie se le permitirá aprovecharse de su propio fraude o sacar partido de su propia injusticia, o fundar demanda alguna sobre su propia iniquidad o adquirir propiedad por su propio crimen. El asesino no recibió su herencia. ${ }^{17}$

13 Esta idea fue, sin embargo, matizada en otras de sus obras. Por ejemplo, García Amado sostiene: "El DWORKIN posterior [a Taking Rights Seriously] es más ambiguo a ese respecto y parece que va plegando velas en lo referido a la fuerza decisoria de los valores morales que son derecho y en lo concerniente a su teoría de la única respuesta correcta (García Amado, 2010, p. 178).

14 Dworkin, op. cit., 1988, p. 74.

15 Ibidem, pp. 47-48.

16 Ibid, p. 49.

17 Ibid, p. 73. 
En Henningsen v. Bloomfield Motors, Inc., sobre la responsabilidad que le corresponde a un fabricante de automóviles por los daños causados en un accidente, el juez sostuvo que:

La libertad de contratación no es una doctrina tan inmutable como para no admitir restricción alguna $[\ldots]$ ¿Hay algún principio que sea más familiar o esté más firmemente integrado en la historia del derecho anglo-norteamericano que la doctrina básica de que los tribunales no se dejarán usar como instrumentos de desigualdad e injusticia?. ${ }^{18}$

Considera que estos casos muestran que es usual para los jueces recurrir a "principios jurídicos", ${ }^{19}$ que plantea como distintos de las reglas por: el carácter de su orientación, pues no aplican en forma de todo o nada; su no aplicabilidad sin excepciones a todos los casos; la imposibilidad de delimitar su contenido haciendo expresas las excepciones que aplican; no establecer las condiciones de aplicación sino apoyar una dirección para alguna decisión; y el hecho de poseer peso o importancia. Así, señala que:

La diferencia entre principios jurídicos y normas jurídicas es una distinción lógica. Ambos conjuntos de estándares apuntan a decisiones particulares referentes a la obligación jurídica en determinadas circunstancias, pero difieren en el carácter de la orientación que dan. Las normas son aplicables a la manera de disyuntivas. Si los hechos que estipula una norma están dados, entonces o bien la norma es válida, en cuyo caso la respuesta que da debe ser aceptada, o bien no lo es, y entonces no aporta nada a la decisión. ${ }^{20}$

En este caso encuentra el autor una primera diferencia entre principios y reglas, que entiende es estructural o lógica, basada en el carácter de la orientación: las normas son disyuntivas, los principios no. Y agrega que, a veces decimos "que nuestro derecho respeta el principio de que nadie puede beneficiarse de su propio delito, pero

$$
\begin{aligned}
& 18 \text { Ibid, p. } 74 . \\
& 19 \text { Ibid, p. } 73 . \\
& 20 \text { Ibid, p. } 75 .
\end{aligned}
$$


no queremos decir con ello que la ley nunca permite que un hombre se beneficie de las injusticias que comete". ${ }^{21}$ Con ello muestra otra distinción entre reglas y principios, pues a diferencia de las reglas, éstos no aplican a todos los casos sin excepción; ni admiten clarificación agregando las excepciones, como en el caso de las reglas, pues no es posible identificar los innumerables casos en que un principio no sería válido, por lo que identificarlos "podría agudizar nuestra percepción del peso del principio [...], pero no nos proporciona un enunciado más completo ni más exacto del mismo". ${ }^{22}$

Luego resalta una característica adicional, consistente en que un principio como el que establece que "(n)adie puede beneficiarse de su propio delito", ${ }^{23}$ no pretende siquiera establecer las condiciones que hacen necesaria su aplicación, sino que enuncia una razón en una dirección, sin exigir una decisión en particular. Si un hombre tiene algo o está a punto de recibirlo, como resultado directo de algo ilegal que hizo para conseguirlo, ésa es una razón que la ley tendrá en cuenta para decidir si debe o no conservarlo. Puede haber otros principios o directrices que apunten en dirección contraria; por ejemplo, una directriz de aseguramiento de derechos, o un principio que limite la pena a lo estipulado por la legislación". ${ }^{24}$ Por tanto, los principios no establecen sus condiciones de aplicación, dejando abierto el ámbito de casos en los que pueden ser aplicables. ${ }^{25}$

Otra característica consiste en el peso o importancia de los principios, por lo que se tienen que comparar cuando varios son aplicables a un caso. Si se da interferencia entre principios, como el de

21 Ibid, pp. 75-76.

22 Ibid, p. 76.

23 Ibid, pp. 75-76.

24 Ibid, pp. 76-77.

25 Esta característica será resaltada de forma detallada por los profesores Manuel Atienza y Juan Ruiz Manero en muchas de sus obras, especialmente en "Las piezas del derecho". 
"la política de protección a los consumidores de automóviles" ${ }^{26}$ y "los principios de libertad de contratación", ${ }^{27}$ quien decide el conflicto tiene que considerar el peso relativo de cada uno; decisión que no es mecánica y puede ser controvertida. Así, a diferencia de los principios, las normas son importantes funcionalmente, sin tener peso. ${ }^{28}$ En cambio, la importancia sistémica de los principios radica en que uno puede sustituir a otro por tener mayor peso.

Los conflictos entre normas se resuelven decidiendo cuál es válida y cuál debe ser abandonada o reformada. Decisión que se da bajo criterios "que trascienden las normas mismas", ${ }^{29}$ como la antigüedad, superioridad o especialidad; incluso los sistemas jurídicos pueden "preferir la norma fundada en los principios más importantes". ${ }^{30} \mathrm{Y}$ agrega lo que parece ser otro criterio de diferenciación: "quien sepa algo del derecho norteamericano sabe que debe tomar el primero de estos enunciados como la expresión de una norma, y el segundo como la de un principio", ${ }^{31}$ señalando que ello puede ser difícil, pues es posible que "no se haya establecido cómo debe operar el estándar", ${ }^{32}$ lo que puede dar lugar a controversias. Remite, como ejemplo, a la cláusula sobre libertad de expresión de la primera enmienda de la Constitución norteamericana que, dice, se puede tomar como norma (toda norma que la contraríe debe declararse inconstitucional) o como principio (mirar el contexto para ver si hay otro principio o directriz que permita la limitación). ${ }^{33}$

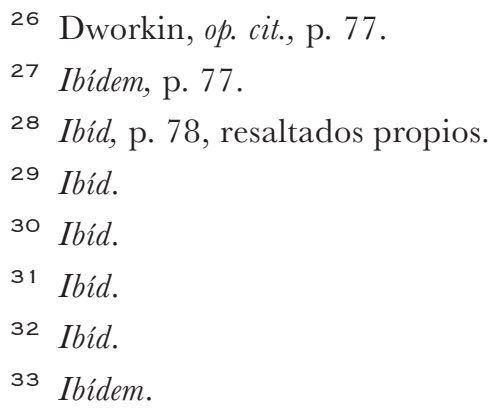


Continúa agregando que, a veces, las normas y los principios desempeñan papeles muy semejantes (función), siendo su diferencia "casi exclusivamente cuestión de forma", ${ }^{34}$ con lo que remite al criterio formal. Sin embargo, al estudiar el Sherman Act, que establece que todo contrato que restrinja el comercio será nulo, muestra cómo, a pesar de las formas, la decisión de la Corte Suprema fue, tratarlo como norma en sentido lógico, pero considerando que funcionaba como principio en sentido sustancial, al contener la palabra "irrazonable", la cual le pareció que lleva a considerar múltiples directrices y principios antes de determinar si alguna restricción es irracional.

Con frecuencia, palabras como "razonable", "negligente", "injusto" y "significativo" cumplen precisamente esta función. Cada uno de estos términos hace que la aplicación de la norma que lo contiene dependa, hasta cierto punto, de principios o directrices que trascienden la norma, y de tal manera hace que ésta se asemeje más a un principio. Pero no la convierten totalmente en un principio, porque incluso el menos restrictivo de estos términos limita el tipo de los otros principios y directrices de los cuales depende la norma. ${ }^{35}$

Sin embargo, esta explicación no deja en claro por qué el uso de este tipo de términos hace que se deba tratar al estándar en cuestión como principio, pues la necesidad de interpretar conceptos vagos es bastante común en derecho.

Además, y en la misma línea de contradicción con lo dicho sobre la distinción como cuestión formal, agrega: "La forma de un estándar no siempre deja en claro si se trata de una norma o de un principio", ${ }^{36}$ aceptando que distinguir principios y reglas no es posible en este terreno. Se sirve de dos ejemplos de enunciado: un testamento no es válido sin la firma de tres testigos, y, un hombre

\footnotetext{
34 Ibid., p. 79.

35 García Amado, op. cit., 2014.

36

Ibídem.
} 
no se puede beneficiar de su propio delito; y reconoce que, en lo formal, no se puede diferenciar entre estas proposiciones. Esta idea será precedente de una práctica común entre quienes defienden la distinción entre reglas y principios: a pesar de proponer diversos criterios de diferenciación, aceptan al final que, por referencia al texto normativo no es posible distinguir entre ellos. ${ }^{37}$

Dworkin da por sentado que ha distinguido los principios como "una clase de estándares aparte, diferente de las normas jurídicas", ${ }^{38}$ y agrega que ello permite ver que estamos rodeados de ellos, y que funcionan con más fuerza y peso en los casos difíciles, pues:

En casos así, los principios desempeñan un papel esencial en los argumentos que fundamentan juicios referentes a determinados derechos y obligaciones jurídicas. Una vez decidido el caso, podemos decir que el fallo crea una norma determinada [...] Pero la norma no existe antes de que el caso haya sido decidido; el tribunal cita principios que justifican la adopción de una norma nueva. ${ }^{39}$

Al distinguirlos, agrega que los principios permiten fundamentar juicios sobre la existencia de derechos y obligaciones jurídicas, y que son argumentos de autoridad para crear tanto nuevas interpretaciones como nuevas normas jurídicas en el sistema, pues dichos principios forman parte del material jurídico existente. ${ }^{40}$

Finalmente, hay que resaltar que la idea clave que le permite entender que los principios hacen parte del derecho radica en su obligatoriedad. "Cuando decimos que un determinado principio es un principio de nuestro derecho, lo que eso quiere decir es que el principio es tal que los funcionarios deben tenerlo en cuenta, si viene al caso, como criterio que les determine a inclinarse en uno $\mathrm{u}$

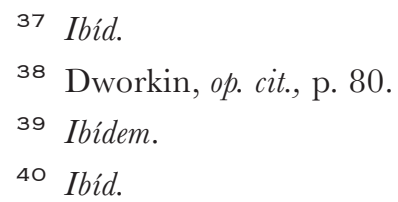


otro sentido", " ${ }^{41}$ pues guían la decisión sin determinarla; muchos ni siquiera especifican los deberes o derechos que de ellos se derivan, o sus condiciones de aplicación; pero no puede obviarse el "importante papel de los principios cuando se trata de llegar a determinadas decisiones jurídicas". ${ }^{42}$

Frente a ello, plantea como posible tratar a los principios jurídicos como normas jurídicas "y decir que algunos principios son obligatorios como derecho y que han de ser tenidos en cuenta por los jueces y juristas que toman decisiones de obligatoriedad jurídica", ${ }^{43}$ o negar que son obligatorios "de la misma manera que lo son algunas normas". ${ }^{44}$ Ello, considera, no es solo una cuestión de palabras sino una elección entre dos puntos de vista de vital importancia para analizar la obligación jurídica: elegir entre dos conceptos de principio, que muestran la diferencia entre tener algo por norma o costumbre, como correr dos kilómetros o el ir al cine una vez por semana, en el ejemplo de Hart, y estar obligado por una norma.

En su opinión, los jueces no actúan en el sentido de tener por norma aplicar ciertos parámetros, sino que existen normas que los obligan. "Si los jueces simplemente "tienen por costumbre" no imponer el cumplimiento de ciertos contratos, por ejemplo, entonces no podemos decir, antes de la decisión, que nadie "tenga derecho" a ese resultado, y esa proposición no puede integrar ninguna justificación que pudiéramos ofrecer para la decisión". ${ }^{45}$ Una de estas versiones, afirma, enfoca los principios como vinculantes, y la otra no. Y ello afecta, incluso determina, "la respuesta que podamos dar a la cuestión de si el juez, en un caso difícil [...] intenta imponer el cum-

\footnotetext{
41 Ibid., p. 77.

42 Ibidem, p. 80.

43 Ibid., p. 81.

44 Ibidem, p. 81.

45 Ibid., p. 82.
} 
plimiento de derechos y deberes preexistentes". ${ }^{46}$ Teniendo como vinculantes a los principios, los jueces aplican "normas jurídicas obligatorias", ${ }^{47}$ imponen el cumplimiento de derechos y deberes jurídicos, sin ejercer discreción.

Como se observa, el criterio fundamental para saber cuándo son obligatorios los principios para los jueces son los derechos preexistentes. Así, refiriéndose a la identificación de los derechos políticos, sostiene Dworkin que no hay procedimiento mecánico para determinarlos, pero, en los casos políticos y jurídicos difíciles, los abogados razonables discrepan, sin que alguno convenza al otro. ${ }^{48}$ Entender que en estos casos no existe derecho alguno presupone una tesis filosófica: "ninguna proposición puede ser verdadera a menos que exista, por lo menos en principio, algún procedimiento para demostrar su verdad de manera tal que cualquier persona racional deba conceder que es válida"; ${ }^{49}$ idea cuyo rechazo considera fundado en buenas razones, especialmente frente a discusiones sobre derechos. En su opinión, es necesario que la teoría política "reconozca que muchas reclamaciones de derecho, incluso algunas muy importantes, no son demostrables, y por consiguiente, ofrecen principios capaces de regir la decisión oficial cuando los derechos son controvertidos". ${ }^{50}$

Es decir, está afirmando la problemática tesis de que es posible encontrar los principios capaces de regir decisiones judiciales, precisamente allí donde no hay acuerdo entre abogados razonables, en casos de reclamaciones de derechos, pues no hay norma que los consagre, y cuya existencia no se puede comprobar intersubjetivamente.

\footnotetext{
46 Ibidem.

47 Ibid.

48 Ibidem, p. 40.

49 Ibidem.

50 Ibid.
} 
En tanto los principios "orientan una decisión en un sentido, aunque no en forma concluyente, y sobreviven intactos aun cuando no prevalezcan"; 51 ello, le parece, no justifica afirmar que los jueces cuando usan principios tienen discreción.

Si un juez cree que los principios que está obligado a reconocer apuntan en una dirección y que los principios que apuntan en otra, si los hay, no tienen el mismo peso, entonces debe decidir de acuerdo con ello, así como debe seguir lo que él cree que es una norma obligatoria. Por cierto que puede equivocarse en su evaluación de los principios, pero puede también equivocarse al juzgar que la norma es obligatoria. ${ }^{52}$

Es paradójico, pero lo importante es que el juez debe creer que está obligado a reconocerlos, y si compiten, debe decidir por los que a él le parezca que pesan más; afirmando que esto mismo se hace con las normas obligatorias. El punto crítico clave, político y de teoría del derecho, es que hay que determinar algún criterio para decidir más o menos objetivamente cuándo una norma o un principio son obligatorios, y esta explicación no es suficiente, a pesar de que, frente a las reglas, sí parece haber algo semejante a tal criterio, el concepto de validez jurídica.

Como se ve, el autor pasa de enfatizar las diferencias entre reglas y principios con base en la lógica, la estructura, la forma, el sentido de la regulación o los efectos, a considerar que los principios son razones a favor de alguna decisión. ${ }^{53}$ Razones que tendrá que tomar en consideración no solo el legislador sino especialmente el juez, a la hora de identificar cuáles son los derechos preexistentes que debe aplicar.

Pero, entender los principios como razones, por su parte, hace que éstos se parezcan mucho a los Topoi: una fuente de argumentos que puede orientar la decisión, o no. Es claro que siempre habrá

\footnotetext{
51 Ibid., p. 89.

52 Ibidem.

53 Ibid., pp. 76-77.
} 
una razón, tal vez varias, detrás de la decisión, y puede darse a este procedimiento cualquier nombre, incluso discrecionalidad; lo relevante es que el fenómeno es, comparativamente considerado, el mismo. Y probablemente tenga las mismas consecuencias en el nivel de lo político.

Por lo dicho, parece que distinguir entre principios y reglas no es un asunto formal, ni estructural ni funcional, ni derivado de alguna característica propia del instrumento normativo sino de otra cosa: cómo deben tomarlos o entenderlos los operadores. Este es un asunto que puede ubicarse en el nivel de la cultura jurídica, o de la pragmática, pero sólo admitiendo, en términos de teoría del derecho, que los elementos del sistema jurídico son aquellos que los operadores jurídicos acepten como tales. Y esto se parece mucho a la tesis positivista de las fuentes sociales del derecho, tan criticada por el autor.

Aunque queda otra alternativa, la de considerar que existe un deber de entender los enunciados normativos a veces como reglas y otras veces como principios. De ser así, tal deber tendría que entenderse como parte de una teoría normativa sobre el derecho; y éstas, en tanto establecen deberes no positivos, son propuestas de mejora del sistema jurídico o cosas semejantes, no explicaciones de cómo es el derecho efectivamente. Y, en tanto teorías normativas, que se supone están fundadas en diversas concepciones morales y políticas, todas se encuentran necesitadas de fundamentación en dichos términos, y no se puede llegar a concluir sin más que, una vez se ofrezca dicha fundamentación, la misma equivale a una afirmación de validez jurídica.

En conclusión, queda claro que para Dworkin la distinción entre tipos de normas o estándares radica en la práctica: si conoces el sistema dado, debes tomar algunos enunciados normativos como norma y otros como principio, sin que se pueda saber de antemano, a partir del texto mismo, cuál es de un tipo y cuál del otro. Es, al final de cuentas, un asunto perteneciente al uso de los enunciados nor- 
mativos, más que a la lógica o función lingüística que éstos puedan desempeñar, o a su estructura.

III. UNA Distinción Estructural, PERO CENTRAda EN EL modo de cumplimiento, desde Robert Alexy

Si Dworkin revivió la discusión teorética sobre principios jurídicos, Robert Alexy profundizó en esta discusión con gran fuerza e impacto, aportándole una amplitud y relevancia especialmente significativas en el derecho constitucional, la teoría del derecho y la argumentación jurídica. Para éste, los principios son:

[...] normas que ordenan que se realice algo en la mayor medida posible, en relación con las posibilidades jurídicas y fácticas. Los principios son, por consiguiente, mandatos de optimización, que se caracterizan porque pueden ser cumplidos en diversos grados y porque la medida ordenada de su cumplimiento no sólo depende de las posibilidades fácticas, sino también de las posibilidades jurídicas. El campo de las posibilidades jurídicas está determinado a través de principios y reglas que juegan en sentido contrario. $^{54}$

Para el autor, los principios son, entonces, normas optimizables, sin que quede del todo claro a qué se refiere exactamente esta idea de optimización o maximización, que desarrolla mediante la ponderación. Por razones de espacio esto no puede discutirse aquí.

Es en la optimización donde encuentra el autor la principal diferencia de principios y reglas, pues éstas, considera, no poseen tal cualidad y, por ello no son objeto de ponderación, mientras que los principios la exigen.

Para explicar los principios jurídicos como normas, acude al enfoque de las razones, según el cual tanto reglas como principios

54 Robert Alexy, Teoría de los derechos fundamentales, Madrid, Centro de Estudios Constitucionales, 1993, p. 14. 
lo son, pues ambas establecen lo que es debido, ${ }^{55}$ pudiendo "ser formulados, con la ayuda de las expresiones deónticas básicas del mandato, el permiso y la prohibición. Los principios, al igual que las reglas, son razones para llevar a cabo juicios concretos de deber ser, aun cuando sean razones de un tipo muy diferente". ${ }^{56}$

Considera esta distinción como de la mayor importancia para una teoría de los derechos fundamentales, pues es la base de la teoría de la fundamentación iusfundamentalmente correcta de los mismos, y clave para la solución de los problemas centrales de la dogmática de los derechos fundamentales.

Sin ella, no puede existir una teoría adecuada de los límites a los derechos fundamentales, ni una teoría satisfactoria de la colisión entre derechos fundamentales y tampoco una teoría suficiente acerca del papel que juegan estos derechos en el sistema jurídico. Es un elemento básico ... de la dogmática de los derechos ... La distinción entre reglas y principios constituye, además, el marco de una teoría normativo-material de los derechos fundamentales y, con ello, un punto de partida para responder a la pregunta acerca de la posibilidad y los límites de la racionalidad en el ámbito de los derechos fundamentales. Por todo esto, la distinción entre reglas y principios es uno de los pilares fundamentales del edificio de la teoría de los derechos fundamentales. ${ }^{57}$

Así, el concepto de principio y su diferencia con las reglas es un elemento crucial para esta teoría; para establecerla, introduce los criterios más comunes de distinción, como la generalidad, su génesis, el contenido valorativo, los tipos de casos a los que aplican, su relevancia en el ordenamiento jurídico, su condición de fundamen-

55 Robert Alexy, Teoría de los derechos fundamentales, $2^{\mathrm{a}}$ ed., Centro de Estudios Políticos y Constitucionales, 2007, p. 65.

56 Robert Alexy, Teoría de La Argumentación Furídica, 2a ed., Manuel Atienza e Isabel Espejo (eds.), Madrid, Centro de Estudios Políticos y Constitucionales, 2007 p. 65.

57 Alexy, op. cit., Teoria... 2007, p. 62. 
to de las reglas, o su relevancia en la argumentación, ${ }^{58}$ y termina por revisar tres criterios: el estructural, que será el eje de análisis en este texto, el papel que juegan como razones, y la generalidad, con base en los cuales, considera, es posible mostrar que hay diferencias cualitativas entre reglas y principios, en tanto éstos son mandatos de optimización, y las reglas no.

Desde el criterio estructural, circunscribe la diferencia entre reglas y principios con base en la exigencia de cumplimiento que deriva de cada uno de ellos:

El punto decisivo para la distinción entre reglas y principios es que los principios son normas que ordenan que se realice algo en la mayor medida posible, en relación con las posibilidades jurídicas y fácticas. Los principios son, por consiguiente, mandatos de optimización, que se caracterizan porque pueden ser cumplidos en diversos grados y porque la medida ordenada de su cumplimiento no sólo depende de las posibilidades fácticas, sino también de las posibilidades jurídicas. El campo de las posibilidades jurídicas está determinado a través de principios y reglas que juegan en sentido contrario. $^{59}$

En tanto mandatos de optimización, los principios son distintos a las reglas, que exigen cumplimiento pleno; por ello, éstas sólo pueden ser cumplidas o incumplidas, al establecer determinaciones en lo posible, fáctica y jurídicamente. En su condición de mandatos de optimización, los principios pueden cumplirse en distintos grados, y como su medida debida de cumplimiento depende de las posibilidades reales y jurídicas, es necesario establecer estas últimas recurriendo a los principios y reglas opuestos.

El carácter de los principios significa que no se trata simplemente de normas vagas, sino que con ellas se plantea una tarea de optimización. Dicha

58 Ibidem, p. 66.

59 Alexy, op. cit, 1993, p. 14; y Teoría..., 2007, p. 67. 
tarea es, en cuanto a la forma, jurídica; en cuanto al fondo, sin embargo, es siempre también moral, a causa de su contenido moral. ${ }^{60}$

En tanto dependientes de las posibilidades, los principios plantean una conexión entre los mandatos del derecho y los de la moral, que se concretizará mediante el recurso a su metodología de aplicación propia, la ponderación. De allí que la distinción entre reglas y principios se pueda identificar mejor en las "colisiones de principios y en los conflictos de reglas", ${ }^{61}$ que muestran el núcleo del problema de la distinción. Pues derivado de su carácter estructural diferenciado, son también diferenciadas las contradicciones entre ellos. Hay contradicción, entiende, cuando dos normas aplicadas independientemente, llevan a resultados incompatibles, por tratarse de dos juicios de deber ser jurídico contradictorios. Los principios entran en tensión recíproca mientras que las reglas entran en conflicto. ${ }^{62}$ En éstos, la validez de alguna de las reglas en conflicto se ve amenazada, lo que no pasa en el caso de los principios.

Presenta dos formas distintas de solucionar dichas contradicciones: el conflicto entre reglas se resuelve al introducir a una de ellas una cláusula de excepción o al declarar la invalidez de una de ellas, expulsándola del ordenamiento jurídico, usando reglas como lex posterior o lex general, o remitiendo a "la importancia de las reglas en conflicto". ${ }^{63}$ En cambio, la colisión de principios se da cuando éstos entran en relación de oposición; ello sucede cuando según uno, algo está permitido, y según el otro lo mismo está prohibido. En estos casos, uno de ellos tiene que ceder ante el otro, sin que ello signifique declarar inválido al que cede, ni haberle introducido una excepción. ${ }^{64}$

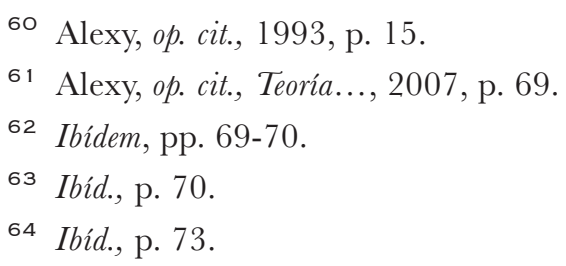


Más bien lo que sucede es que, bajo ciertas circunstancias, uno de los principios precede al otro. Bajo otras circunstancias, la pregunta acerca de cuál es el principio que prevalece puede ser solucionada de manera inversa. Esto es lo que se quiere decir cuando se afirma que en los casos concretos los principios tienen diferente peso y que prima el principio con mayor peso. ${ }^{65}$

Luego enuncia como ejemplos de solución de colisiones, las numerosas "ponderaciones de bienes", ${ }^{66}$ del Tribunal Constitucional Federal, en las que encuentra la estructura de las soluciones de colisiones, que resume en la "ley de la colisión", ${ }^{67}$ y, la concepción del resultado de la ponderación como una norma adscrita de derecho fundamental (Alexy, 2007, p. 71), para las cuales, si se van a entender como normas de derecho fundamental, debe ser posible aducir una "fundamentación iusfundamental correcta". ${ }^{68}$

Desde la óptica de la argumentación, reglas y principios poseen distinto carácter prima facie, pues al ordenar "que algo debe ser realizado en la mayor medida posible, teniendo en cuenta las posibilidades jurídicas y fácticas", ${ }^{69}$ los principios no contienen mandatos definitivos sino sólo prima facie. ${ }^{70}$ De allí que el que un principio valga para un caso no significa que ello tenga validez como resultado definitivo; ellos presentan razones que pueden ser desplazadas por otras, no determinan cómo se resuelve la relación entre una razón y su opuesta, pues carecen de "contenido de determinación con respecto a los principios contrapuestos y las posibilidades fácticas", ${ }^{71}$ mientras que las reglas sí.

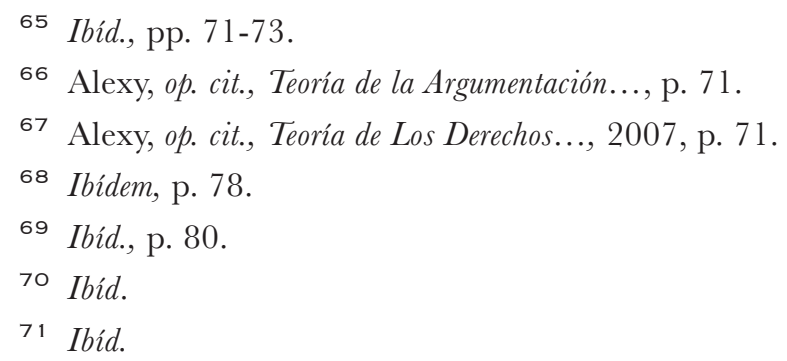


Considera erróneo el asumir que "todos los principios tienen un mismo carácter prima facie y todas las reglas un mismo carácter definitivo", ${ }^{72}$ tal como lo percibe en el modelo "simple" de Dworkin, según el cual cuando las reglas tienen validez, son aplicables de una manera todo-o-nada, mientras que los principios "sólo contienen una razón que indica una dirección pero que no tiene como consecuencia necesariamente una determinada decisión". ${ }^{73}$ Considera que hace falta un modelo más diferenciado, dentro del cual hay que "mantener el diferente carácter prima facie de las reglas y los principios". ${ }^{74}$ Frente a las reglas, pues éstas pueden ser excepcionadas mediante un principio, lo que hará que pierdan su carácter definitivo, adquiriendo un carácter prima facie, "básicamente distinto del de los principios". ${ }^{75}$ Para desplazar la regla se necesita que un principio opuesto tenga mayor peso en el caso concreto, y se deben desplazar "los principios que establecen que deben cumplirse las reglas que son impuestas por una autoridad legitimada para ello y que no es posible apartarse sin fundamento de una práctica que proviene de la tradición"; ${ }^{76}$ es decir, también hay que desplazar los principios formales.

Pero las reglas no tienen el mismo carácter prima facie de los principios, ni siquiera cuando su carácter definitivo ha sido debilitado; y el reforzar el carácter prima facie de los principios, "introduciendo una carga de argumentación en favor de determinados principios o determinados tipos de principios", ${ }^{77}$ tampoco permite que éstos tengan el mismo carácter prima facie de las reglas, siendo el carácter prima facie de ambas no equiparable.

\footnotetext{
72 Ibid., p. 70.

73 Ibid., p. 80.

74 Ibid.

75 Ibid., p. 81.

76 Ibid.

77 Ibid.
} 
Esto muestra, en su opinión, que reglas y principios son razones de distinto tipo; razones, siempre prima facie los principios, definitivas las reglas, salvo que se haya establecido una excepción. Y ambas, además, pueden ser tanto razones para la acción como "razones para normas"; 78 tanto reglas como principios pueden ser razones tanto para normas universales como para normas individuales (juicios concretos de deber ser jurídico).

En tanto razones, en el nivel constitucional son prevalentes los principios, pues las reglas son solo determinaciones de éstos. "Una decisión fundamental de un constituyente no puede ser otra cosa que una decisión a favor de un determinado principio o de determinados principios". ${ }^{79}$ Esto es muy problemático, pues quiere decir que los principios, además de ser entendidos como instrumentos normativos autónomos dentro del sistema jurídico, tienen la capacidad adicional de ser la fuente del resto de las normas, las reglas, que quedan así reducidas a ser meras concreciones de principios, lo que permitirá obviar las decisiones del constituyente o el legislador, y decidir a favor de los principios. Por lo mismo, y en el caso de todas las reglas que no contienen en su enunciado el principio que les sirve de fundamento, debería entenderse, al parecer, que el mismo está contenido en algún principio implícito.

Frente a la distinción entre principios y policies, sostiene que ambos tienen propiedades lógicas distintas, por lo que conviene distinguirlos, pues no es necesario vincular los principios únicamente con los derechos individuales sino también con bienes colectivos. Sin embargo, en toda la propuesta se puede ver cómo el autor asimila los principios con las directrices, especialmente al entenderlos como mandatos de optimización. Así lo da a entender, por ejemplo,

\footnotetext{
78 Ibid., p. 82.

79 Ibid., p. 148.
} 
cuando afirma que, detrás de una norma que establece un fin hay un principio que es idéntico al fin mismo. ${ }^{80}$

Por otro lado, al distinguir entre principios escritos y no escritos, sostiene, respecto de los principios no derivables de enunciados del sistema jurídico, que se trata de normas "surgidas naturalmente", y que, al poderse contraponer a las "creadas", queda claro que los principios no requieren ser establecidos de forma explícita sino que pueden, también, ser "derivados de una tradición de expedición detallada de normas y, de decisiones judiciales que, por lo general, son expresión de concepciones difundidas acerca de cómo debe ser el derecho". ${ }^{81}$

El punto crítico que merece ser resaltado es que, frente a las normas que establecen fines, y que pueden considerarse como directrices, cabe perfectamente la posibilidad de cumplimiento en más o en menos, es decir, optimizando o maximizando. Ello es mucho más discutible en materia de los principios distintos a las directrices, vinculados a derechos. Si se presta atención a la ponderación, se verá que siempre, como resultado de ella, y como criterio para resolver el caso que dio origen a la colisión de principios, aparece una regla que será aplicada en todo o nada al caso en cuestión, ${ }^{82}$ sin que quepa optimización o modalidad graduable de cumplimiento, ni en el procedimiento de ponderación entre principios, que da lugar, luego de establecidas las condiciones de precedencia condicionada de la ley de colisión, ${ }^{83}$ a la regla que servirá de referente para resolver el caso, ni en la aplicación de la regla misma.

Adicionalmente, se hace relevante el tratamiento que el autor da a esta situación, pues muestra dos cosas: que hay un fin que el Estado persigue; y que hay un principio en el cual el Estado fundamenta

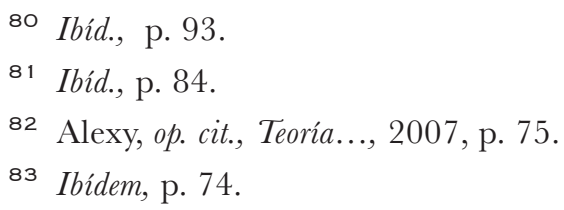


la persecución de dicho fin. Esto plantea la cuestión de saber dónde encontrar sustento a la afirmación de que hay un principio idéntico al fin expresamente perseguido, y que es precisamente el que el Estado ha elegido para fundamentarlo. Pero, más allá de ello, el punto aquí importante es el abanico de cuestiones que deja abiertas: ¿es el principio que fundamenta un fin, en todos los casos, idéntico al fin de que se trata?, ¿esto hay que presuponerlo?, ¿`su existencia exige una fundamentación iusfundamental correcta? o ¿al menos alguna fundamentación?

De allí que sólo queda concluir que cada norma constitucional que ordena la obtención de fines está fundamentadas en un principio idéntico al fin perseguido, con lo cual ya no hace falta hablar de normas que establecen fines, pues lo importante en ellas es que son equivalentes a principios; y, por ser principios/espejo de normas que establecen fines, es fácil utilizarlos como ejemplo de procesos de optimización de las necesidades fácticas, usando para ello los sub-principios de necesidad e idoneidad. Éstos, sin embargo, no parecen tan fácilmente utilizables cuando la colisión creada se presenta entre principios que no consagran fines, sino que establecen derechos fundamentales.

Es decir, bajo la óptica de esta propuesta, entendiendo los principios como mandatos de optimización, los únicos que podrían ser calificados como principios serían las directrices, o policies, pues dado que de la ponderación resultante de una colisión entre dos principios, se deriva una nueva regla que será la que hay que subsumir para resolver el caso, entonces no es cierto que los principios se cumplan de forma graduable, en más o en menos, tal como lo afirman críticamente los profesores Manuel Atienza y Juan Ruiz Manero, ${ }^{84}$ siendo sólo posible ello frente a las directrices. A lo sumo, se puede aceptar que los principios se cumplen a veces sí y a

84 Atienza y Ruiz Manero, op. cit., 2004, p. 168. 
veces no, tal como se deriva de la ponderación; pero esto ya no es gradualidad, ni maximización u optimización.

Al contrario, con esta teoría se proponen criterios de inaplicación puntual, caso a caso, de los principios, al menos de los que, según Dworkin son principios en sentido estricto, referidos a valores o a derechos individuales. En estos casos, tales principios no pueden cumplirse en más o en menos; se cumplen de forma definitiva, una vez establecida mediante ponderación la regla aplicable al caso, resultante de la colisión de principios, según esta misma propuesta.

De allí que lo que el autor llama optimización no pasa en este caso de ser la inaplicación de una disposición explícita y aplicable, a favor de la aplicación de un principio, no necesariamente explícito y sólo eventualmente aplicable, en tanto su aplicabilidad depende de la pre-comprensión que haga el aplicador sobre el problema jurídico a ser resuelto en la situación de tensión o conflicto de que se trate.

Lo dicho nos lleva a concluir que el concepto mismo de principio, como mandato de optimización, resulta problemático en el caso específico de los que Dworkin reconoce como principios en sentido estricto. De allí que se pueda afirmar que esta propuesta parece estar diseñada para su aplicación solo al caso de las policies.

Además, al aceptar que hay principios no expresos, lleva a la paradójica tesis de que hay elementos dentro de los sistemas jurídicos (los principios) que, siguiendo el concepto jurídico de validez (que es uno de los conceptos positivistas que acepta el autor) no están dentro del sistema, pero que siempre que se necesiten pueden ser traídos a éste y hacérseles funcionar como un elemento más dentro del mismo, siendo capaces de derrotar a los otros elementos, que sí hacen parte del sistema (las reglas, e incluso las constitucionales).

Así, al aceptar la existencia de principios no explícitos, es posible concluir que, primero, los principios son diferenciables de las disposiciones autoritativas establecidas de forma expresa en la Constitución; segundo, que, en virtud de ello, se trata en todo caso de 
interpretaciones, y que, para llegar a ser tal cosa, es necesario que el intérprete u operador jurídico haga una de dos cosas:

- que le agregue interpretativamente, a la norma derivada de una disposición, una cláusula que la haga siempre derrotable frente a principios y valores contrapuestos que pueden tener mayor peso en el caso concreto. ${ }^{85}$

- que, en caso de no haber disposición a interpretar, busque dentro de la tradición algún principio que ya, por no estar ligado a ninguna disposición, no requiera tal cláusula, y se haga aplicable directamente.

De esta forma queda claro que los principios no son elementos que integran el ordenamiento jurídico, sino que, en pos de aceptarlos, podría decirse que se trata de argumentos que admite la tradición jurídica o que son una forma de interpretar los contenidos de las disposiciones. Pero de entenderlos así, queda abierta la cuestión del por qué dichos argumentos poseen la fuerza jurídica suficiente para derrotar a los contenidos de las disposiciones constitucionales.

A partir de las definiciones anteriores, se deduce que el concepto de principio como mandato de optimización es algo que hay que suponer, puesto que esta teoría no ofrece razón o evidencia alguna para demostrar que los principios están, como elementos autónomos, dentro del sistema jurídico. No se puede olvidar que el principio como tal surge después del proceso interpretativo mediante el cual se agrega la cláusula que hace derrotable a la norma en cuestión, al agregarle la salvedad de que lo regulado funciona tal como está regulado, siempre que no precedan los principios o valores opuestos. De esta forma, entonces, aparece claro que la relativización respecto de las posibilidades jurídicas es algo que sólo aparece en escena una vez se ha interpretado la disposición en cuestión, o

85 Alexy, op. cit., Teoría de los derechos..., 2007, pp. 116-117. 
incluso ninguna disposición en el caso de los principios implícitos, en el sentido de agregar tal cláusula que la convierte en principio.

Como se observa, la distinción estructural, que entiende el autor como la principal, posee muchas inconsistencias. Primero, por partir de un concepto de principio bastante problemático, el de mandato de optimización, que se ha mostrado cómo sólo es aplicable a las directrices, pero no lo es de forma tan sencilla cuando se trata de explicar los otros tipos de principios. Segundo, porque el carácter de la distinción que el autor denomina estructural no parece recaer precisamente sobre la estructura de la norma jurídica, sino en su forma de vinculación. Tercero, porque de lo anterior se deriva, en esta propuesta, la idea de que los conflictos entre principios son distintos a los de las reglas, y se resuelven también distinto, recurriendo a la ponderación. Cuarto, porque lo que se presenta como una necesidad derivada de una colisión entre principios, ponderar, resulta ser un requisito previo: los principios exigen ponderación porque en su misma definición exigen ser confrontados con principios y reglas opuestos. Y para enfrentar una solución normativamente establecida en disposiciones autoritativas, es requisito previo y necesario haber interpretado los hechos de manera que se plantee dicha necesidad de no aplicar directamente sino poner a competir la solución establecida con un principio contradictorio. Quinto. porque las distintas funciones argumentativas o justificativas que cumplen los principios y las reglas, según esta propuesta, depende directamente de interpretaciones previas sobre la ponderación.

IV. UNA GENUINA DISTINCIÓN ESTRUCTURAL Y SUS PROBLEMAS. Manuel Atienza y Juan Ruiz Manero

Por su parte, y en el ámbito hispanohablante, el trabajo de los profesores Atienza y Ruiz Manero es paradigmático, entre muchas otras, sobre la idea de principios. Para esta revisión se partirá de "Las piezas del derecho", incluyendo en lo pertinente, otras obras relaciona- 
das; su relevancia principal radica, especialmente, en su cuidadoso desarrollo de la distinción estructural entre principios y reglas jurídicas, y las críticas que han hecho a las propuestas antes revisadas.

Los principios jurídicos son, para estos autores, un tipo especial de enunciados jurídicos, con carácter normativo; que expresan normas. Entre éstos distinguen las reglas, de acción y de fin; y los principios, en sentido estricto y directrices o normas programáticas.

Para el análisis estructural de los principios, retoman el esquema de Alchourrón y Bulygin, ${ }^{86}$ quienes definen las normas jurídicas como una estructura condicional que relaciona un caso, conjunto de propiedades, con una solución o calificación normativa de una determinada conducta, y analizan desde allí tanto los principios como las reglas. Sobre el concepto de principio, Ruiz Manero sostiene que:

[...] los principios en sentido estricto pueden ser vistos como normas categóricas (en el sentido de von Wright, esto es, que no establecen condiciones de aplicación distintas de las que se derivan de su contenido) que generan deberes meramente prima facie; si, en relación con un cierto caso, deseamos saber si ese deber prima facie deviene deber concluyente hemos de añadir al antecedente, constituido por la existencia de una posibilidad de realizar (u omitir) la acción modalizada deónticamente en el consecuente, una cláusula de "a menos que": el deber prima facie deviene deber concluyente siempre que sobre el caso en cuestión no incida otro principio que tenga, en relación con él, un peso mayor y que, por ello, derrote, en relación con el caso, al principio bajo consideración. De esta forma, los principios en sentido estricto pueden ser vistos como normas hipotéticas (asimismo en el sentido de von Wright, esto es, que establecen condiciones de aplicación adicionales a aquellas que se derivan de su contenido) que generan deberes concluyentes. La diferencia con las reglas (que también establecen condiciones de aplicación adicionales a aquellas que se derivan de su contenido) es que, en el caso de las reglas, esas condiciones de aplicación están determinadas (o, lo que es lo mismo, se presentan, como preferíamos decir en

86 Carlos Alchourrón y Eugenio Bulygin, Introducción a la metodología de las ciencias jurídicas y sociales, Buenos Aires, Astrea, 1974. 
Las piezas, en forma cerrada), mientras que en el caso de los principios en sentido estricto tales condiciones de aplicación están indeterminadas (o, lo que es lo mismo, se presentan, como preferíamos decir en Las Piezas, en forma abierta). ${ }^{87}$

El hecho de que sus condiciones de aplicación estén configuradas de forma abierta, hace de los principios un tipo especial de norma en cuyo antecedente:

[...] no se contiene otra cosa sino que se dé una oportunidad de realizar la acción modalizada deónticamente en el consecuente. Y esta modalización deóntica no pretende, como en el caso de las reglas, ser concluyente, sino meramente prima facie, por la simple razón de que en muchas ocasiones en que se da la oportunidad de realizar la acción ordenada por un cierto principio, se da también la oportunidad de realizar la acción, incompatible con la anterior, ordenada por otro principio. Siendo así las cosas, los principios no pueden, por su propia configuración, eximir a sus destinatarios de la tarea de determinar si en unas determinadas condiciones o circunstancias lo ordenado por un cierto principio debe, o no, prevalecer sobre lo ordenado por otro principio que resulte concurrente. ${ }^{88}$

Lo que significa que, a diferencia de las reglas, la deliberación aparece en este caso como ineludible, y por ello se tiene que recurrir a la ponderación judicial que permita formular una nueva regla que determine, esa sí con carácter concluyente, la prevalencia de un principio sobre otro en ciertas circunstancias determinadas. De allí concluyen que los principios en sentido estricto, las directrices y las reglas, siempre pueden entenderse como normas con la estructura condicional caso/solución. Sin embargo, a pesar de ello, se diferencian en la forma de configurar los casos. Las reglas configuran los casos de forma cerrada y los principios de manera abierta. En las reglas están definidas desde el enunciado normativo las condiciones de aplicación o caso y la solución; por su parte, en los principios

87 Ruiz Manero, Juan, Principios, objetivos y derechos. Otra vuelta de tuerca, en Doxa, 2005, vol. 28.

88 Ibidem. 
las condiciones de aplicación se encuentran establecidas de forma abierta.

[...] mientras que en las reglas las propiedades que conforman el caso constituyen un conjunto finito y cerrado, en los principios no puede formularse una lista cerrada de las mismas: no se trata sólo de que las propiedades que constituyen las condiciones de aplicación tengan una periferia mayor o menor de vaguedad, sino de que tales condiciones no se encuentran siquiera genéricamente determinadas. ${ }^{89}$

Dentro de los principios, las directrices configuran de forma abierta tanto las condiciones de aplicación como el modelo de conducta prescrito. ${ }^{90}$ Las directrices dejan

[...] abiertas sus condiciones de aplicación, ordenan -o permiten- procurar alcanzar un estado de cosas en la mayor medida posible. Las directrices se asemejan a las reglas de fin en que dejan abierta la configuración del modelo de conducta idóneo para lograr el estado de cosas ordenado, pero se diferencian de éstas en que tal estado de cosas aparece configurado asimismo de forma abierta (debe lograrse en la mayor medida posible, en conjunción con otros objetivos que también se ordena maximizar). Ello, naturalmente, implica que las directrices admiten modalidades graduables de cumplimiento. ${ }^{91}$

A su vez, las directrices se diferencian de los principios en sentido estricto en que éstos, una vez establecida la preferencia en caso de conflicto operan como las reglas, pues configuran de forma cerrada la conducta regulada o solución, mientras que las directrices lo hacen de forma abierta. De esta forma,

la distinción más importante es la que media entre principios en sentido estricto y directrices o normas programáticas. Los primeros establecen que, salvo que en el caso de que se trate incida(n) otro(s) principio(s) que tengan un mayor peso, debe - o puede- $^{-}$seguirse un determinado modelo

89 Atienza y Ruiz Manero, op. cit., 2004, p. 10.

90 Ibid.

91 Ibid., p. 168. 
de conducta que aparece configurado, al modo de las reglas de acción, de forma cerrada (lo que implica que, en los casos en que prevalece un determinado principio frente a otros eventualmente concurrentes, éste exige un cumplimiento pleno. $^{92}$

Además, las directrices no ordenan o prohíben acciones sino la consecución de algún objetivo; ello marca otra diferencia entre éstas y los principios en sentido estricto. La consecución del objetivo debe darse según su concurrencia con otros fines y objetivos; por tanto, ello sólo puede darse en la mayor medida posible, de forma graduable. De allí que frente a las directrices lo procedente no es la ponderación sino la articulación: “... a diferencia de lo que ocurre con los principios en sentido estricto, en la conducta gobernada por directrices no se trata de determinar la prevalencia de una u otra en relación con un determinado caso, sino de articular políticas capaces de lograr, en el mayor grado posible, la consecución conjunta de todos estos objetivos". ${ }^{93}$

Por su parte, las reglas de fin, afirman, funcionan de forma semejante a las directrices, pues ordenan la obtención de fines, con la diferencia de que las reglas de fin regulan la consecución del fin propuesto de forma plena. Operan de forma distinta a las directrices, cuyo cumplimiento es graduable. "Lo que las reglas de fin no cierran es el modelo de conducta prescrito, pues ordenan simplemente el logro de un determinado estado de cosas, sin prejuzgar las acciones que constituyen medios idóneos para ello", ${ }^{94}$ dejando en ocasiones a sus destinatarios un margen de discreción que no existe en el caso de las reglas de acción". ${ }^{95}$

En conclusión, desde el enfoque estructural, los autores aceptan que reglas y principios comparten la misma estructura condicional,

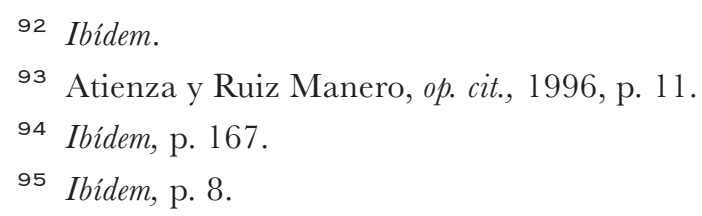


que es la de caso/solución; pero, simultáneamente son distintos, por la distinta forma en que los diversos tipos de enunciados normativos regulan los elementos dentro de dicha estructura. ${ }^{96}$ Así sostienen que:

Desde el punto de vista estructural, todos ellos pueden reducirse a una forma condicional en la que el antecedente (supuesto de hecho, condiciones de aplicación o caso) está constituido por un determinado estado de cosas, y el consecuente (la consecuencia jurídica o solución normativa) lo integra una acción (en el caso de los principios en sentido estricto o en el de las reglas de acción) o bien un estado de cosas (si se trata de directrices o de reglas de fin) modalizados mediante operadores de mandato ... o bien mediante el operador facultativo. ${ }^{97}$

En conclusión, los autores consideran que la diferencia entre reglas y principios radica, no en la estructura condicional en sí misma, que comparten, sino en la forma como los elementos funcionan dentro de ella, siendo lo más relevante, como factor de distinción, la manera en que está configurado el caso o antecedente. Y es especialmente importante, por la diferencia que plantean respecto de lo propuesto por Alexy, la distinción que proponen entre el modo de cumplimiento de las directrices, que es graduable, y el de los principios en sentido estricto, que sostienen es del tipo de todo-o-nada.

En conclusión, y según lo visto, para los profesores Atienza y Ruiz Manero existe tanto coincidencia como diferencia en el plano estructural entre reglas y principios. La coincidencia radica, para esta concepción, en que ambos tipos de enunciados pueden ser explicados remitiendo a una misma estructura: caso/solución, mediados por un operador deóntico; la diferencia principal radica en la manera cómo está regulado el caso en tales tipos de enunciado, estando abierto este elemento en los principios y cerrado en las reglas.

\footnotetext{
96 Ruiz Manero, op. cit., 2005.

97 Atienza y Ruiz Manero, op. cit., 1996, p. 167.
} 
Una revisión crítica de la estructura categorial propuesta permite observar algunas cuestiones relevantes, como: que el caso o las condiciones de aplicación se encuentren configuradas de forma abierta en todos los principios, significa que este tipo de enunciados no expresan las circunstancias, condiciones o actividades mediante las cuales, en el ordenamiento jurídico, se considera procedente el deber de activación de una solución o consecuencia jurídica; de lo que se deriva que una variedad de acciones y circunstancias no específicamente reguladas o determinadas como un supuesto de hecho, pueden dar lugar a la solución o consecuencia, en tanto la principal característica de los principios consiste en que en su antecedente no se contiene otra cosa sino "que haya una oportunidad de realizar la conducta ordenada en el consecuente", 98 es decir, que carece de un antecedente normativo.

Por tanto, el carácter abierto del caso en los principios plantea un problema a la hora de determinar su aplicabilidad a las situaciones fácticas, de modo que éstos pueden eventualmente resultar aplicables a un número indeterminado de casos particulares y viceversa: dado un caso concreto será posible o no que un principio sea aplicable y que, además, concurra con otros principios o reglas también potencialmente aplicables al mismo. Este resultado es ya, por lo menos, problemático, en tanto implica que el operador jurídico tendrá, en los principios así definidos, una posibilidad de aplicación de consecuencias normativas a cualquier circunstancia que eventualmente encaje o pueda hacerlo, en el contenido del principio, sin que los destinatarios de dichas normas puedan saber de antemano el contenido de la conducta eventualmente obligatoria, prohibida o permitida. Ello parecería exigir una operación de complementación normativa que, en esta propuesta, queda directamente en manos del juez.

98 Sobre este punto, se puede consultar: Ruiz Manero, 2005, p. 345; Atienza y Ruiz Manero, 1996; Atienza y Ruiz Manero, 2004 
Por otra parte, los principios consagran una solución, una conducta regulada de forma cerrada (para los principios en sentido estricto) o un estado de cosas regulado de forma abierta (para las directrices). Y, en tanto esta acción o estado de cosas está deónticamente modalizado, se puede afirmar que se trata de una obligación, una prohibición o un permiso. De esta forma, los principios en sentido estricto, al configurar la solución de forma cerrada, no admiten modalidades graduables de cumplimiento, el cual debe darse de forma plena; al contrario de las directrices o mandatos de optimización que, para los autores y de acuerdo con Alexy, sí las admiten.

Lo anterior por el hecho de que las directrices se pueden cumplir en más o en menos, mientras que los principios en sentido estricto se cumplen de forma total. ${ }^{99}$

Finalmente, es relevante presentar una idea hasta ahora no discutida, sobre la forma de identificar un principio jurídico. Ello, como se ha visto, tiene relación con su estructura, pero también con otro asunto: para que los principios puedan ser ponderados, es necesaria en esta propuesta, de forma similar a lo propuesto por Alexy, la inclusión de una cláusula adicional a lo contenido en el enunciado normativo autoritativo. Así, además de la caracterización estructural de los principios, al estar éstos definidos con sus condiciones de aplicación abiertas, se hace necesaria una interpretación que lleva a ponderar, pues tales normas no excluyen, sino que demandan, la deliberación. Ello parece implicar, el entender el principio no ya como una estructura sino como una norma (interpretada) dotada de una cláusula adicional, que no se suele encontrar expresamente regulada y supone una salvedad: "salvo que en el caso de que se trate incida(n) otro(s) principio(s) que tengan un mayor peso". ${ }^{100}$ Esto significa que, previa a la identificación estructural de una

\footnotetext{
99 Atienza y Ruiz Manero, op. cit., 1996, p. 10.

100 Ibidem, p. 168.
} 
norma, que permitiera determinar si se trata de una regla o un principio, es necesario realizar una interpretación de la misma, para incluir dicha cláusula, según la cual es procedente la ponderación en todos los casos en los que incidan principios de mayor peso. Así, en cualquier situación de regulación jurídica, donde es posible que coexistan principios contrarios que puedan llegar a tener un peso mayor, habrá que determinar la necesidad de la ponderación y, a partir de ella, construir la solución del caso.

Como se observa, la comprensión de un enunciado normativo como un principio implica dos momentos: primero, hay un principio cuando estemos frente a un enunciado que configure de forma abierta sus condiciones de aplicación y establezca de forma cerrada una solución; ello significa que, estructuralmente, principio es una norma que sólo modaliza deónticamente una consecuencia jurídica sin establecer un supuesto de hecho.

Segundo, concebir un enunciado normativo como un principio, implica para los autores concebirlo como contentivo de una cláusula no expresa, sino que deviene resultado de una cierta interpretación, que indique que, salvo mayor peso de otro(s) principio(s) concurrente(s) y contrario(s), debe aplicarse lo contenido en la consecuencia del principio en cuestión. De esta manera, entonces, esta segunda manera de concebir el principio ya no resulta ser estructural sino interpretativa, pues implica abordar la semántica del principio, al agregarle interpretativamente al enunciado normativo una hipótesis no contemplada en él y que cambia su contenido normativo, pues conlleva a determinarlo judicialmente mediante la ponderación.

\section{v. Conclusiones}

Para finalizar, y adicional a las consideraciones críticas que se han ido introduciendo en cada apartado de este escrito, resulta importante retomar algunas de las razones por las cuales se puede afirmar 
que, con base en los autores estudiados, la distinción estructural entre principios y reglas no se sostiene.

Por una parte, la propia idea de principios resulta problemática. En Dworkin, puesto que éste los entiende como el resultado que se deriva de una concepción del sistema que incluya tanto los elementos morales como los políticos del sistema social, y que integre además todas las reglas y precedentes jurídicos vigentes, así como lo que se puede esperar que se aplicarán en coherencia con el mismo.

La cuestión fundamental aquí, independientemente del hecho de que tales concepciones son construcciones interpretativas y, por tanto, dependientes de las habilidades intelectuales, morales y argumentativas del intérprete, es que en la medida en que los principios dependen de tales construcciones, entonces la afirmación de su diferencia estructural con las reglas, entendiendo por ésta una diferencia en los términos de su estructura lingüística o normativa, es imposible de sostener.

Una crítica adicional tiene que ver con la idea de Dworkin de que hay derechos preexistentes al sistema de normas de derecho positivo; frente a ello hay que reconocer que la teoría política o moral admite el reconocimiento de reclamaciones de derechos que no son demostrables, pero que pueden estar fundados en buenas razones. El terreno político y moral son precisamente los escenarios en que se da la lucha social entre ideologías y visiones de vida buena que se pretende sean implementadas como proyecto social. Y que de ello se derive que, "por consiguiente", tales reclamaciones ofrecen principios capaces de regir (que pueden ser utilizados en) las decisiones jurídicas oficiales en los casos difíciles, es también aceptable, aunque no se vea la relación consecuencial entre lo uno y lo otro, pues no existe; se trata de una relación meramente contingente. Pero, sostener que de ello se derive algún tipo de deber de las autoridades decisoras para utilizar esos principios - o cualquier otra clase de fuente extrajurídica o no demostrable que pudiera ser aplicable a, o regir la, decisión- es totalmente cuestionable. Una vez más nos 
encontramos con un uso falaz del discurso, que dice una cosa aparentando decir otra.

Desde la perspectiva de Alexy, el concepto de principio también presenta el problema de ser categorizado como parte de las normas que integran el sistema jurídico, pero siendo distintos de las reglas. Éstos poseen un componente sustantivo, que es moral $\mathrm{y}$, adicionalmente, son distintos de los enunciados normativos autoritativamente establecidos. Con ello, se llega en esta propuesta a que los principios se construyen interpretativamente, bien sea a partir de enunciados autoritativos o no. Pero lo relevante es que los mismos son el resultado de un proceso de interpretación en el que se hace necesario agregar una cláusula que haga derrotable lo contenido en la regulación, al establecer su obligatoriedad como dependiente del resultado de su confrontación o pesaje con otros principios o valores opuestos. De esta manera, entonces, se hace imposible la distinción estructural, en los términos enunciados, pues el principio sólo aparece después del ejercicio interpretativo que le agrega dicha cláusula y lo torna en algo ponderable.

Por el lado de los profesores Atienza y Ruiz Manero, el análisis lleva a conclusiones semejantes a las hechas frente a Alexy. Ello, en la medida en que estos autores entienden que el principio exige agregarle una cláusula adicional que supone una salvedad, consistente en que lo regulado deviene aplicable salvo que, en el caso en cuestión, incidan otros principios con mayor peso. Con ello se llega a igual resultado: los principios sólo aparecen con posterioridad al proceso interpretativo. Por ello, los importantes aportes que en el terreno de la dimensión estructural realizan estos autores se debilitan pues sólo serán relevantes después de construido interpretativamente el principio, y con ello, la distinción estructural deviene como consecuencia de la interpretación, careciendo de autonomía.

Por otra parte, y como resultado de lo dicho, así como los principios son algo que se construye interpretativa y argumentativamente, bien a partir de enunciados normativos expresos o no, la distinción 
estructural que nos ofrecen los distintos autores es también un producto de la construcción interpretativa y argumentativa. Con ello, entonces, queda sentado que, como elementos autónomos del sistema jurídico, los principios no son estructuralmente diferenciables de las reglas jurídicas.

\section{Fuentes}

Alchourrón, Carlos E, and Bulygin, Eugenio, Introducción a La Metodología de Las Ciencias Juridicas $Y$ Sociales, Buenos Aires, Astrea de Rodolfo Depalma y hnos., 1974.

Alexy, Robert, Teoría de los derechos fundamentales, Madrid, Centro de estudios Constitucionales, 1993.

- Teoría de la argumentación jurídica, Atienza, Manuel y Espejo, Isabel (eds.), $2^{\mathrm{a}}$ ed., Madrid, Centro de Estudios Políticos y Constitucionales, 2007.

- Teoría de los derechos fundamentales, Carlos Bernal Pulido (ed.), $2^{\circ}$ ed., Madrid, Centro de Estudios Políticos y Constitucionales, 2007.

- Tres escritos sobre los derechos fundamentales y la teoría de los principios, Bogotá, Universidad Externado de Colombia, 2003.

Atienza, Manuel y Ruiz Manero, Juan, Las Piezas del Derecho, Barcelona, Ariel, 1996.

Atienza, Manuel, El derecho como argumentación, Barcelona, Ariel, 2006.

Dworkin, R. M., Los derechos en serio, Barcelona, Ariel, 2012.

Dworkin, Ronald, El imperio de la justicia, Barcelona, Gedisa, 1988.

- Los derechos en serio, Barcelona, Ariel, 1984.

- , Los derechos en serio, 2a ed., Barcelona, Ariel, 1989.

García Amado, Juan Antonio, "Neoconstitucionalismo, ponderaciones y respuestas más o menos correctas", en El derecho y sus circunstancias, Bogotá, Universidad Externado de Colombia, 2010, pp. 169-206.

— "Pidiendo el Principio. Dworkin y la teoría del derecho en serio", en línea, disponibleen: <www.garciamado.es $>$. 
Hart, H. L. A., The Concept of Law, 2a ed., Oxford, Clarendon Press, 1994. Melero de la Torre, Mariano, Dworkin y sus críticos, Madrid, Tirant lo Blanch, 2012.

Ripstein, Arthur, "Dworkin”, en Ronald Dworkin, 2007.

Ruiz Manero, Juan, "Principios, objetivos y derechos. Otra vuelta de tuerca," Doxa, Cuadernos de filosofia del derecho, 28 (2005), pp. 341-65. 